\title{
MOM Investment and Its Development Prospects in China
}

\author{
Xiaolong Jia ${ }^{1, \mathrm{a}}$, Qiyue Cheng ${ }^{1, \mathrm{~b}}$, Zihao $\mathrm{Pu}^{1, \mathrm{c}^{*}}$, Ling Chen $^{1, \mathrm{~d}}$, Yuchen $\mathrm{Ma}^{1, \mathrm{e}}$ \\ ${ }^{I}$ Zhonghui Lianyang (Zhuhai Hengqin) Asset Management Co., Ltd., Chengdu, China, 610000a \\ a1599058869@qq.com \\ bqiyue625@outlook.com \\ c*thoupu@163.com \\ d1021102171@qq.com \\ e754996030@qq.com
}

\begin{abstract}
With the increasing importance of risk hedging in the financial market, MOM funds have become one of the best choices for investors. This article carefully summarizes the development background and four-step operation process of MOM funds. In view of the similarity between FOF and MOM, this paper groundbreakingly elaborates the differences between them from eight aspects. At the same time, based on China's national conditions, this paper interpreted the MOM policy, and analysed the opportunities and challenges of China's MOM development in the future.
\end{abstract}

Keywords: MOM, Fund manager, asset control, investment style, investment ability, market environment, regulatory policy

\section{MOM投资及其在中国发展前景}

贾小龙 ${ }^{1, a}$ 程启玥 ${ }^{1, b}$ 蒲梓昊 ${ }^{1,0 *}$ 陈玲 $^{1, d}$ 马宇 辰 $^{1, e}$

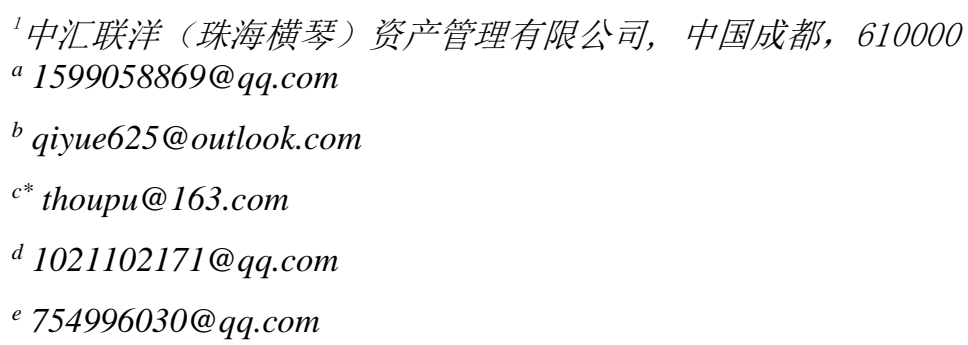

摘要

随着风险对冲的重要性在金融市场日益增加，MOM基金成为了投资者最优的选择之一。本文细致总结了MOM基 金的发展背景及四步运作流程。鉴于FOF与MOM具有一定的相似度，本文开创性地从8大方面详细阐述了二者之 间的区别。同时，基于中国国情，对MOM政策进行了解读，并分析了未来中国MOM发展的机遇和挑战。

关键词：MOM，基金经理，资产控制，投资风格，投资能力，市场环境，监管政策 


\section{1. 介绍}

\section{1. MOM的基本介绍}

MOM 投资模式为管理人的管理人基金 (Manager of Mangers) 模式。1980 年美国罗素资产管理公司（ Russe11 Investment）提出 MOM 基金这一概念。MOM 的基金管理人通过长期跟踪、研究基金经理投资过 程，集合各领域优秀基金经理，以投资子账户委托 形式让他们负责投资管理的一种投资模式，是基金 经理的投资组合。

1940 年代, Alfred Winslow Jones 在美国创立 了第一支对冲基金，他使用股票多空对冲的方式来 降低熊市的风险, 这也就是 “对冲” 两字的由来。 Evans and Archer 在 1968 年就指出通过持有 10 支 股票就基本能消除掉大多数种类的风险 [1]。但是基 金特有的高风险性并且缺乏透明度促进了 FOF 和 MOM 的诞生。1970 年, Adam Street, Crossroads, Harbour Vest 为一些大的机构投资者同时进入多个 PE 基金提供了高效渠道。1980 年美国罗素资产管理 公司（Russel1 Investment）发行了第一支 MOM 基 金。Li Yuxiang 等将经理的个人特征界定为年龄、 性别、实战经验、投资经验、职业经历和教育。他 认为基金经理的某些个人特性对基金业绩有重要影 响[2]。Hongde Ai 等运用 logistics 回归发现从业 时间长, 或毕业于中国名校, 或获得经济学学位的 基金经理倾向于稳健的投资风格，拥有金融分析师 资格的基金经理更倾向于价值型投资 [3]。 Chevalier 和 Ellison 将基金经理的个人特征界定为 经理所毕业大学的 SAT 分数, 是否获得 MBA 学位, 经理的年龄和任期。他们认为, 经理毕业于 SAT 分 数较高的大学, 具有更高的智力 或者直接受益于更 好的教育 [4]。每类资产和策略都有适合自己的市场 环境, 每位基金经理都有自己擅长的领域和周期, MOM 产品以多元化资产配置、多元化投资风格和多元 化子管理人来优化收益风险比, 穿越牛熊, 跨越周 期。

JAMES M. PARK 和 JEREMY C. STAUM 基于 CTAs, 对冲基金和 NYSE 股票得出结论， “过于多样化” 不 会导致多样化收益的减少 [5]。Stephen J. Brown 等 在 2003 年针对 FOF 提出一种新的费用机制以吸引更 多投资者 [6], Andrew Ang 等在 2008 年通过使用资 产配置的概念来估计基金基准分布的特征, 认为为 FOF 支出的高费率是值得的 [7]。而 MOM 相对于 FOF 更低的费率更是其较大的优势。

\section{2. $M O M$ 的核心理念}

MOM是具有双层管理人结构的组合类产品, 产品 整体层面和子基金层面分别由不同的管理者管理。 其核心理念是在大类资产配置的基础上，分析基金 经理的投资理念和管理水平，挑选出投资风格契合、 管理能力突出的子管理人, 构建组合并动态管理,
从而降低系统性风险和策略失效风险, 获取长期稳 定的超额收益。

\section{3. MOM基金发展现状}

MOM 业绩相对稳定，因此受到大型企业年金、养 老基金、慈善基金以及保险资金等大型机构的亲崃, 是全球资管市场的重要组成部分之一。从全球来看, 根据 Lipper 的统计数据, MOM 基金市场规模从 2000 年底的 3298 亿美元快速增长至 2017 年底的 12229 亿 美元，在欧美主流市场资产管理规模占有一席之地。 市场上发行 MOM 产品的普遍为富达、罗素、贝莱德 等大公司。在美国，根据 Cerulli Associates 统计 的数据, 从 2000 年到 2006 年, 美国 MOM 的数量从 2440 亿美元增长到 6390 亿美元。截止 2019 年底, 美国公募 MOM 达到 3 万亿美元, 占美国全部共同基 金的 $27 \%$ 。从资产及策略类别看, 在美国股票型 MOM 产品数量占绝对优势, 混合型和债券型 MOM 产品数 量相当。

而 MOM 产品在我国的发展处于初期。2019 年 12 月 6 日，证监会发布《证券期货经营机构管理人中 管理人（MOM）产品指引（试行）》, 国内首份正式 的 MOM 操作指引发布。此后, 华夏、招商、创䠇合 信、建信、鹏华等 5 家基金公司上报了公募 MOM 产品, 并于 2020 年 2 月 18 日收到第一次反馈意见。

\section{MOM运作流程}

\section{1. 设定投资目标}

首先确定 MOM 母基金的目标，才能做出正确的 战略配置，常见的投资目标有：

- 养老金计划：资金的储备和投资是为了支付 未来养老金，投资计划需是长期稳健型;

- 险资投资：在未来不确定的时间点支付设定 好的保险赔偿金，对投资风险和流动性有较高要求;

- 匹配负债：对于有负债的机构，投资目标之 一是匹配负债;

- 产品定位：为满足不同客户的投资需求，产 品定位在低风险、可持续获取稳定收益。

明确投资目标后，选择对应的业绩比较基准以 反应组合的表现是否达预期，比如宽基指数、同类 组合业绩的比较基准等, 选择的对比标的需能反映 投资目标。

基于投资目标, 从四个维度考量, 即收益目标、 风险目标、流动性目标和投资限制; 设定对比指标, 在此基础上对宏观经济和市场环境做出判断。

高收益、高流动性和低风险这三个因素无法同 时满足，是投资中经典的 “不可能三角” ，因此根 据投资目标来平衡三者的重要性。资产定价模型 
（CAMP）是最经典的管理收益风险模型，由于真实 市场环境的不确定因素较多, 不少研究员开发出了 多种模型。

制定收益目标时需要考虑到通货膨胀、费用和 税收等因素。受通胀影响, 名义收益率和实际收益 率会留有差别，且部分底层资产具有抗通胀性；费 用包括交易费用，运营费用、管理费用及销售费用 等，投资人的目标收益率是扣除费用和成本后的收 益率; 不同的资产，做交易时的税率也不一样，属 于成本的一部分。

风险方面, 除了投资策略本身的回撤风险, 还 有三个主要影响因素, 规模、期限和流动性; 一般 情况下, 投资模越大, 投资期限越长, 流动性需求 越低, 其承受风险能力越强。主要的几大类风险及 风控措施分别为: 风险

- 市场风险：分散化投资，对冲策略均可降低

- 信用风险: CDS 对冲、不完全对冲可平滑信 用风险

- 流动性风险：不同周期的产品和策略组合

- 运营风险：尽职调查

在制定投资目标中, 需考虑到真实投资环境中 的投资限制, 比如:

- 投资期限：投资期限的长短会影响投资标的 的选择及风险水平;

- 监管政策：对储备金及投资标的份额的限制, 如：信托每年分配资产不可超过 10\%;

流动性: 流动性的强弱直接影响资产类别的选 择。

\section{收益目标}

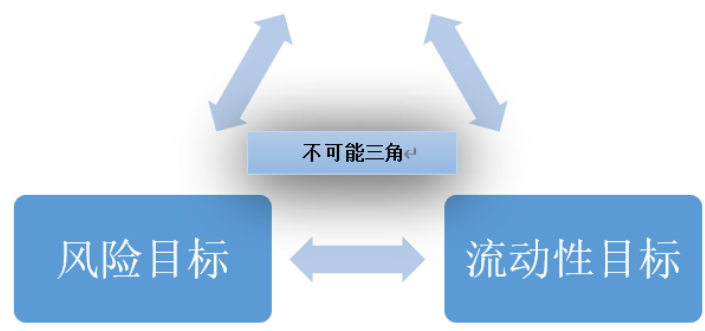

图 1 不可能三角

\section{2. 分析市场环境}

根据宏观经济数据、市场情绪、技术指标等判 断当前市场的周期及未来走向, 并结合各大类资产
的长期收益率和波动率，运用资产配置模型对各类 投资策略进行研究确定。

权益市场的涨跌幅和成交量相辅相成，判断市 场多空情绪, 结合 GDP、CPI、PMI 和产出缺口等经 济数据的变动率, 确定市场所处的经济周期。在特 定的宏观背景下, 通过资产多元化和策略多元化来 制定配置方案。主要的几大类资产有股票、债券、 商品、外汇、房地产、现金等。基于不同资产的收 益和风险属性，在不同的经济周期内，各类资产的 表现不相同。

美林时钟模型是从宏观经济出发，对当前的经 济周期进行刻画，并给出经济周期与大类资产的映 射关系，从而分析得到最优的资产配置类别。当经 济处于恢复上升期时，货币政策倾向于宽松，公司 业绩显著提升, 股票对经济上行的弹性最大，优选 资产有股票和债券。当经济处于过热期时，较高的 $\mathrm{GDP}$ 推动 $\mathrm{CPI}$, 商品类资产与 $\mathrm{CPI}$ 正相关, 优选资产 有商品和股票。当经济上涨有压力, 处于滞涨期时, 央行通常会采取货币紧缩政策来控制通胀，最先反 应出来的是 GDP 指标遇拐点, 而 CPI 指标依然较高, 货币政策持续紧缩，从而提高了货币资产的收益水 平，相比股票、债券和大宗商品而言，货币类资产 更具有投资价值, 其次是商品。当经济开始下滑趋 势已定, 处于衰退期时, GDP 和 CPI 均处于较低位置, 为刺激经济, 央行又开始采取货币宽松政策, 而此 时市场低迷, 需求疲软, 缺乏上行动力, 而债券中 的长端利率由于处于利率曲线高点的成本优势, 成 为优质投资资产。

经典的马科维茨均值-方差模型 [8]则是以不同 的权重将风险资产进行组合，得到一系列具有不同 期望收益和标准差的风险资产组合。

除此之外，风险平价模型是对组合中的各类资 产分配相等的风险权重, 使各资产为组合所贡献风 险相等, 从风险分配的角度来得到资产配置的组合 权重, 从而科学有效的分散风险, 以实现长期稳定 的回报。

\section{3. 选择基金经理}

在选择各类资产的资管人时，以定量为基础结 合定性的研究，并长期跟踪基金经理业绩，篮选出 能够持续创造超额收益且风格稳定的标的基金经理, 主要从五个维度一一基金公司概况、基金经理个人 能力及品行、投研团队实力、基金经理过往产品业 绩及风险收益特征、业绩归因一一进行综合考量, 按照策略和风格对基金经理分类储备。

\subsection{1. 投资能力分析}

通过定量的方法分析基金经理的投研能力，主 要考量投资风格和历史业绩两个方面。通过投资风 格分析来评估基金经理的风格偏好，母基金管理人 
可据此相对准确测算每一次风格调整带来的收益和 风险, 并持续追踪子管理人是否出现风格漂移; 通 过历史业绩归因分析来评估基金经理过往产品的资 产配置能力、风险收益水平、进攻防守能力和盈利 持续性等。

\subsubsection{1. 投资风格.}

由于 MOM 基金是笁选管理人做组合，因此基金 经理的投资风格和风格稳定性是构建 MOM 组合的最 重要的因素。通过 Barra 模型将组合收益分解到公 共因子收益上及行业收益上，衡量该基金经理的收 益来源在各类风格及各行业中的暴露度, 在时间序 列上分解管理人风格配置和行业配置的风险及收益 率，判断基金经理的投资风格是否随时间的变动而 漂移。

\subsubsection{2. 历史业绩.}

关于基金经理的历史业绩，本文认为可以从三 方面分析。一是资产配置能力, 二是风险收益水平, 三是进攻防守能力。

资产配置能力是指通过行业偏离度和持仓集中 度衡量基金管理人的配置决策的合理性和在不同周 期下投资策略的风险性，也在一定程度上评估管理 人的投资风格。

而评价风险收益的指标有夏普比率、特雷纳指 数、索提诺指数、M2 测度、詹森 $\mathrm{a}$ 、最大回、 Calmar 比率等 (举例说明) :

- Calmar 比率: 收益与最大回撤的比值, Calmar 比率数值越大，基金的业绩表现越好。反之， 基金的业绩表现越差。

$$
\text { Calmar Ratio }=\frac{\text { 年化收益率 }}{\text { 最大回撤 }}
$$

- 夏普比率: 收益率与波动率的比值, 夏普率 越大, 表明在承担同等风险的情况下, 投资组合获 得的收益越大。

$$
\text { Sharp Ratio }=\frac{\mathrm{R}_{\mathrm{p}}-\mathrm{R}_{\mathrm{f}}}{\delta_{\mathrm{p}}}
$$

其中 $R_{p}$ ：投资组合的实际收益率; $R_{f}$ ：无风险 利率; $\delta_{\mathrm{p}}$ : 投资组合的标准差。

基金经理的进攻/防守能力可以通过所管理产品 在市场上行/下行期间的累计收益率来衡量。

$$
\begin{aligned}
& \text { 上行区间收益率 }=\left[\prod_{R_{m, t \geq 0}}^{T_{1}}\left(1+R_{p, t}\right)\right]^{\frac{1}{T_{1}}}-1 \\
& \text { 下行区间收益率 }=\left[\prod_{R_{m, t<0}}^{T_{2}}\left(1+R_{p, t}\right)\right]^{\frac{1}{T_{2}}}-1
\end{aligned}
$$

产品的上行区间收益率越高，基金经理在上行 市场中能更好的抓住投资机会，进攻能力越强；如 果产品的下行区间收益率越高，基金经理能更好的 抵抗市场下行风险，防守能力更强。

\subsection{2. 非投资能力分析}

\subsubsection{1. 组织结构合理性.}

从公司战略、规模大小、企业文化、激励制度 等多方面综合评估一家投资公司架构的合理性。公 司结构需与其战略和规模相匹配，公司各部门是否 具备相应的专业度, 并且不同部门之间是否有效沟 通协作, 公司是否有与时俱进、变革创新能力, 员 工薪酬方案是否具有良性竞争特点。

\subsubsection{2. 运营及合规的风控水平.}

风控贯穿了投资交易的每一个环节，一家合格 的投资机构应制定清晰、合规的交易规则，并具备 完善的中后台部门来支持前端部门工作，在产品运 营中风控措施及合规机制应环环相扣，以确保交易 执行的有效性和产品管理的合规性。

\subsubsection{3. 运营及合规的风控水平.}

借鉴罗素公司开发的的基金经理评价体系，可 从四个维度考量:

- 团队管理能力: 基金经理及其团队 的专业水平、员工素质和持续发展能力等因素 可间接衡量管理人是否可获取稳定收益。

自我认知：管理人应客观面对自己 成功和失败的投资决策，清晰的了解自己的能 力圈。

- 目标：有目标的基金经理更专注于 自己的投资理念，并严格执行投资决策，并且 不断精进自己的策略。

- 发展性: 追求发展并不断学习的基 金经理会随着市场的变化做出适应性改变, 可 以持续创造收益。

\section{4. 构建投资组合}

根据对每一位基金经理的综合评估，在不同资 产类别和不同策略类别中分别选出优秀的管理人, 构建一个全方位的投资管理人才库，并持续跟踪。

根据投资目标，在配置好的各大类资产上，从 管理人储备池中挑选对应的基金经理，进行资金分 配，构建投资组合; 并对组合进行动态管理，动态 组合管理主要分两部分一一动态组合调整和动态风 险控制。 


\section{- 动态组合调整}

母基金管理人通过动态大类资产择时、动态风 险预算管理和动态子管理人业绩归因及风格分析等 方式, 及时调整组合中各资产类别或策略类别的权 重。母基金管理人每日关注各类策略的净值表现及 监控子管理人的资金配置, 每月对策略月度收益进 行归因测试并观察投资风格与资产类别是否偏离并 与子管理人沟通策略是否发生调整, 每季度对市场 环境进行判断, 分析各类策略的收益贡献情况, 比 较各子管理人的风险回报评级, 并做出决策是否增 减策略或重新调整子管理人的资金配置等。

\section{- 动态风险控制}

从最初的产品设计到投后的动态管理, 风控环 节贯穿整个流程。从最初的产品设计到投后的动态 管理，风控环节贯穿整个流程。

产品设计：投资风险前置化, 需确保产品流动 性和风险收益目标合理。

市场分析：分析市场风险并及时预警。

管理人笁选：评估管理人风险管理能力, 考核 交易策略及风控机制。

构建组合：设定各类资产或策略的风险预算值， 确保整体风险始终低于预算总限额。

投后管理：对业绩与风险分别归因，比较风险 预算与实际组合风险, 确保组合风险低于预算限额。

\section{MOM与FOF的差异}

MOM 和 FOF 都是投资工具的一种，基于分散化投 资的原则——分散资产类别, 分散管理人一一降低 组合的波动性, 但是 MOM 和 FOF 也存在着以下八个 方面的主要差异（表 1)：

\section{表 1 FOF 与 MOM 的区别}

\begin{tabular}{|c|c|c|}
\hline & MOM & FOF \\
\hline $\begin{array}{l}\text { 研究 } \\
\text { 方法 }\end{array}$ & $\begin{array}{l}\text { 更专注于投资管理人 } \\
\text { 研究, 通过历史业绩 } \\
\text { 考量基金经理的投资 } \\
\text { 能力和非投资能力 }\end{array}$ & $\begin{array}{l}\text { 更专注于产品研究, } \\
\text { 通过历史业绩对管理 } \\
\text { 人过往表现评估 }\end{array}$ \\
\hline $\begin{array}{l}\text { 投资 } \\
\text { 方式 }\end{array}$ & $\begin{array}{l}\text { 通过对基金经理的研 } \\
\text { 究，选择优秀管理人 } \\
\text { 做组合，投资的是 } \\
\text { “人” , 并以投资顾 } \\
\text { 问的形势与母基金管 } \\
\text { 理人合作 }\end{array}$ & $\begin{array}{l}\text { 通过对基金产品的研 } \\
\text { 究, 选择符合母基金 } \\
\text { 经理投资决策的标准 } \\
\text { 化基金产品构建组 } \\
\text { 合, 通过认/申购基 } \\
\text { 金产品进行投资 }\end{array}$ \\
\hline $\begin{array}{l}\text { 策略 } \\
\text { 适应 } \\
\text { 性 }\end{array}$ & $\begin{array}{l}\text { 交易指令要经过母基 } \\
\text { 金管理人的系统审 } \\
\text { 核, 交易速度受到影 } \\
\text { 响, 因此不太适合高 } \\
\text { 频交易。 }\end{array}$ & $\begin{array}{l}\text { 可容纳一些特定策略 } \\
\text { (如大宗交易、定增 } \\
\text { 等) 需要较大的投资 } \\
\text { 规模, 可以由多只母 } \\
\text { 基金投向同一只子基 } \\
\text { 金, 从而发挥规模效 } \\
\text { 应。 }\end{array}$ \\
\hline
\end{tabular}

\begin{tabular}{|c|c|c|}
\hline $\begin{array}{l}\text { 费率 } \\
\text { 结构 }\end{array}$ & $\begin{array}{l}\text { 通过投顾模式运作, } \\
\text { 单层收费 }\end{array}$ & $\begin{array}{l}\text { 投资市场上现有的基 } \\
\text { 金产品, 双重收费 }\end{array}$ \\
\hline $\begin{array}{l}\text { 组合 } \\
\text { 调整 } \\
\text { 方式 }\end{array}$ & $\begin{array}{l}\text { 调入/调出子管理 } \\
\text { 人, 增加/减少子管 } \\
\text { 理人长户资金规模 }\end{array}$ & $\begin{array}{l}\text { 调入/调出基金产 } \\
\text { 品, 追加/赎回基金 } \\
\text { 产品份额 }\end{array}$ \\
\hline $\begin{array}{l}\text { 风控 } \\
\text { 环节 }\end{array}$ & $\begin{array}{l}\text { 由母基金经理统一下 } \\
\text { 单, 统一管理, 风险 } \\
\text { 更好监控, 仓位管理 } \\
\text { 更灵活, 更便于对子 } \\
\text { 管理人进行业绩评估 }\end{array}$ & $\begin{array}{l}\text { 母基金管理人可能看 } \\
\text { 不到子层的实际持 } \\
\text { 仓, 并且基金产品有 } \\
\text { 申购赎回时间的限 } \\
\text { 定, 母层管理人无法 } \\
\text { 根据对市场的判断及 } \\
\text { 时做出调整 }\end{array}$ \\
\hline $\begin{array}{l}\text { 投资 } \\
\text { 独立 } \\
\text { 性 }\end{array}$ & $\begin{array}{l}\text { 当多个子管理人的投 } \\
\text { 资决策有冲突时 (一 } \\
\text { 位管理人看多一只股 } \\
\text { 票, 另一位管理人看 } \\
\text { 空, 产品内不能进行 } \\
\text { 反向交易), 子管理 } \\
\text { 人需接受母层管理人 } \\
\text { 调节 }\end{array}$ & $\begin{array}{l}\text { 投资的是独立的基金 } \\
\text { 产品, 不会有交易冲 } \\
\text { 突 }\end{array}$ \\
\hline $\begin{array}{l}\text { 效率 } \\
\text { 及规 } \\
\text { 模 }\end{array}$ & $\begin{array}{l}\text { 不需单独成立子基 } \\
\text { 金, 运营成本较低, } \\
\text { 产品最低规模限制的 } \\
\text { 要求相对较低; 但如 } \\
\text { 果更换投资顾问, 手 } \\
\text { 续繁琐 }\end{array}$ & $\begin{array}{l}\text { 申购/赎回产品, 运 } \\
\text { 营成本和时间成本都 } \\
\text { 相比 MOM 较高, 但投 } \\
\text { 资策略改变时, 直接 } \\
\text { 更换产品, 无需过多 } \\
\text { 手续 }\end{array}$ \\
\hline
\end{tabular}

\section{MOM在我国的发展前景}

\section{1. MOM的监管政策}

2019 年 12 月 06 日证监会出台《证券期货经营 机构管理人中管理人（MOM）产品指引（试行）》 $[9]$ ，该《指引》首次肯定了母管理人聘请多投资顾 问进行投资管理运作的模式，首次明确了 “管理人 中管理人” 业务范畴和监管框架, 为 MOM 业务健康 有序稳定发展提供了有效的规范依据, 给传统 MOM 业务发展提供了一条创新之路。

该《指引》主要是为了引入资本市场中长期资 金, 为 MOM 运作进行规范。MOM 在我国定义如下:

（一）管理人按照本指引要求委托两个或者两 个以上符合条件的第三方资产管理机构（即投资顾 问）就资产管理产品的部分或者全部资产提供投资 建议; 
（二）根据资产配置需要将资产管理产品的资 产划分成两个或者两个以上资产单元，每一个资产 单元按规定单独开立证券期货账户。

\subsection{1母基金管理人角度分析}

该规定适用于证券公司、公募基金管理人、期 货公司及前述机构依法设立的从事私募资产管理业 务的子公司。对于银行 MOM 以及信托 MOM, 需要等待 银保监会另行制定细则。

\subsection{2母基金管理人和投资顾问权责角度分析}

投资顾问在过去被称为 “子管理人”，如今投 资顾问起到的角色只是为特定子资产单位 “提供投 资建议”，也就是说投资顾问只提供意见，母基金 管理人负责真正的负责 “投资决策” 及 “交易执 行”。管理人应当与投资顾问签订投资顾问协议, 协议中应当进一步明确管理人与投资顾问双方的权 利义务。

\subsection{3产品形式上分析}

MOM 产品既可以通过公开募集方式设立又可以通 过非公开募集方式设立。托管人与投资顾问不得为 同一机构, 不得相互出资或者持有股份。

\section{1. 4更换投资顾问的操作分析}

公开募集 MOM 产品变更投资顾问的，应当依法 履行向中国证监会申请基金产品变更注册等法定程 序。非公开募集 MOM 产品变更投资顾问的, 应当按 照中国证券投资基金业协会的规定履行产品变更备 案等程序，并抄报中国证监会相关派出机构。

\section{1.5 关联交易的角度分析}

监管要求中管理人、投资顾问不得以 MOM 产品 的资金与关联方进行不正当交易、利益输送、内幕 交易和操纵市场。因为实际上投资顾问关联方的交 易指令不会受到母基金管理人的监管, 所以关联交 易比较难控制, 正常的关联交易是被允许的, 重大 关联交易则应当进行披露。关于 MOM 产品是否可以 再投资其他资管产品, 《指引》中并未明确禁止。

\subsection{MOM的机遇与挑战}

MOM 具有 “低波动、中高收益、稳定可持续” 的 风险收益特征, 可以更好的满足机构投资者需求, 尤其是对保险、养老、银行理财和基金会等, MOM 模 式是非常好的选择。

对标美国市场，MOM 基金从成立，便得到市场认 可并发展迅速, 从市场表现来看, 在美国的近十几 年年度策略统计中, 每连续 6 年的统计排名中 MOM 都
排第一。虽然 MOM 模式在海外已发展较为成熟, 但 在国内尚处于初始阶段, 近年来由于部分类别的资 产受到政策限制，投资者面临投资难、资产荒等问 题，权益市场受到越来越多投资者认可，而 MOM 基 金的特性可同时达到机构投资者和个人投资者的期 望, 将会成为市场上主流权益产品之一。

由于产品是母基金经理统一管理, 子基金经理 只提供投资建议，因此母基金经理需有一套完善的 技术系统, 包括交易执行、风控系统、市场跟踪、 择时等; 除此之外, 母管理人需在产品整体风控与 子管理人策略自由度之间做出平衡, 在产品总持仓 不违背合同约定的情况下给予子管理人尽可能多的 操作空间。

MOM 的核心理念是通过分散策略和分散管理人来 降低整体风险, 但在实际情况中, 会遇到共性风险 集中的问题，即两个或多个子管理人/策略的投资逻 辑共线性较大; 一些有效的投资思路广为人知, 如 估值、动量、规模等效应，不同的基金经理个性化 的投资思路中可能存在共性部分，从而选出的标的 高度相关，一般情况下可能影响不大，但如果遇到 金融危机，共性化因素凸显强烈，与其对应的风险 也急剧上升。

\section{5. 结论}

至今，在全球没有关于 MOM 的论文的情况下， 本文细致总结了从对冲基金到 MOM 基金的发展过程 及 MOM 基金的全球发展现状。在 MOM 的运作流程方 面，本文总结为设定投资目标、分析市场环境、选 择基金经理和构建投资组合这四步。在筛选基金经 理的过程中, 本文从投资能力和非投资能力及其包 含的五个方面进行了分析。除此之外, 本文从研究 方法、投资方法等 8 个方面详细阐述了二者之间的 区别。FOF 更注重产品的特性, MOM 更关注单个管理 人的相对优势。

在中国权益市场的重要性增加的情况下, MOM 基 金将成为投资者对风险规避的最优选择之一。共性 风险集中和监管政策障碍也是 MOM 基金潜在的困难。 综上所述, 本文对我国尚处于起步阶段的 MOM 基金 市场给出了一定的指导。

\section{REFERENCES}

[1] Evans, J., \& Archer, S. (1968) Diversification and the Reduction of Dispersion: An Empirical Analysis. The Journal of Finance, 23(5), 761-767.

[2] Yuxiang, Li, Cheng Jian, and Peng Cong. (2006) The Research of the Manager's Personal Characteristic to the Fund Achievement [J]. Value Engineering 12.

[3] Hongde Ai, Cong Liu. (2008) Personal characteristics of fund managers and fund investment style $[\mathrm{J}]$. Financial and Trade Economy, 12:26-31. 
[4] Chevalier J., Ellison G. (1999) Career Concerns of Mutual Fund Managers[J]. Quarterly Journal of Economics, 114(2):389-432,

[5] Park J M, Staum J C. (1998) Diversification: How Much is Enough?[J]. Available at SSRN 85428.

[6] Brown, Stephen J., William N. Goetzmann, and Bing Liang. (2005) Fees on fees in funds of funds. The World Of Hedge Funds: Characteristics and Analysis. pp 141-160,

[7] Ang A, Rhodes-Kropf M, Zhao R. (2008) Do fundsof-funds deserve their fees-on-fees?[R]. National Bureau of Economic Research. No. w13944.

[8] Markowitz, Harry. (2012) Portfolio Selection. Journal of Finance 7.1:77-91.

[9] China Securities Regulatory Commission. (2019) Guidelines for the Manager (MOM) Products of the Managers of Securities and Futures Business Institutions (for Trial Implementation)

http://www.csrc.gov.cn/pub/zjhpublic/zjh/201902/ P020190222553003938822.pdf 